
2 Research Square
Preprints are preliminary reports that have not undergone peer review.
They should not be considered conclusive, used to inform clinical practice, or referenced by the media as validated information.

\title{
The Impact of Selective Survival on Sex Disparities in Stroke Incidence: Evaluating Bias using a Simulation Model
}

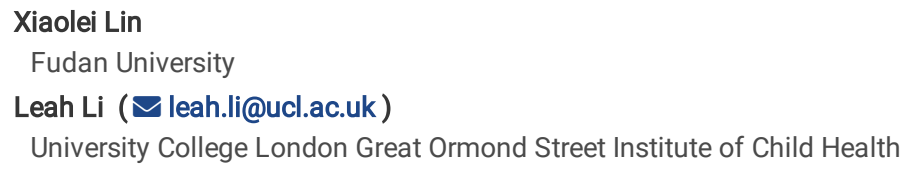

Xiaolei Lin

Fudan University

Leah Li ( $\nabla$ leah.li@ucl.ac.uk)

University College London Great Ormond Street Institute of Child Health

\section{Research Article}

Keywords: Selective survival, survivor bias, sex disparities, stroke incidence rate, risk factors for survival

Posted Date: January 21st, 2022

DOI: https://doi.org/10.21203/rs.3.rs-1163954/v1

License: () (7) This work is licensed under a Creative Commons Attribution 4.0 International License. Read Full License 


\section{Abstract}

Background: Males tend to have higher stroke incidence than females. The difference attenuates (or even reverses) in late-life. Using a simulation study, we assessed how selective survival impacted on age attenuation of the male-female difference in stroke incidence.

Methods: We simulated a birth cohort of 50,000 males and 50,000 females. The distributions of survival and first stroke incident were calibrated from published data. We generated an unobserved construct $U$ (i.e. risk factors for incident stroke and/or survival) and considered four causal scenarios based on the sex-specific association between $U$ and survival. To assess the bias under each scenario, we compared male-female difference in stroke incidence from 45 to $95 y$ with pre-specified value (5 per 1000 person-year).

Results: Under all causal scenarios, male-female difference in stroke incidence was close to pre-specified value from mid-adulthood but decreased (thus bias increased) at older ages. There was only a small increase in bias when $U$ did not affect survival (Scenario $A$ ). When $U$ had a direct effect on survival, the difference decreased (thus bias increased) rapidly, reversed from $95 \mathrm{y}$ when $\mathrm{U}$ affected survival with a similar magnitude for males and females (Scenario B: hazard ratio $(H R)=1.5)$. The difference reversed earlier when the effect of $U$ on survival was greater for males than females: at $85 \mathrm{y}(\mathrm{Scenario} C$ : $\mathrm{HR}=1.5 \mathrm{vs} 1.0)$ and $80 \mathrm{y}$ (Scenario D: HR=2.5 vs 1.5).

Conclusions: Males had higher stroke incidence overall, but the male-female difference attenuated with age when there existed unobserved common risk factors for stroke and survival. The survivor bias was greater when their association with survival differed by sex.

\section{Introduction}

Stroke is currently the second leading cause of death and is responsible for approximately $11 \%$ of the total deaths globally(1). During most of the adult life, stroke incidence is higher for males compared to females. The reasons for the sex disparities in stroke incidence are not fully understood. Differential distributions of stroke risk factors in males and females may partly account for the disparities. For example, high blood pressure, atrial fibrillation and type 2 diabetes have been found to be more common in males than in females(2). Smoking and heavy drinking are also more prevalent among males(3). Moreover, associations between risk factors and stroke may differ by sex $(4,5)$.

However, the magnitude of the male-female difference (or ratio) in stroke incidence attenuates in late life $(6,7)$. A meta-analysis of 59 studies worldwide showed that the age-adjusted stroke incidence rate was $33 \%$ higher in males than in females. The male-to-female incidence rate ratio peaked in the $65-74 y$ age band, reduced between 75-84y, and attenuated further thereafter(8).

The sex difference in stroke may eventually reverse the direction at older ages. In a population-based study in Sweden, females had $60 \%$ lower stroke incidence rate between $55-65 y$, but $50 \%$ higher rate after $75 y$ than males(9). In the UK Oxford Vascular Study, the incidence rate of ischemic stroke was lower between 55-75y, but higher after 85y in females compared to males in the same age groups(10). In the US REGARDS (Reasons for Geographic and Racial Differences in Stroke) cohort, stroke incidence was four times greater in males than in females aged from 45 to $54 y$. The male-to-female incidence rate ratio attenuated at older ages and was lower in males by $85 \mathrm{y}(11)$.

This attenuation (or reversal) of the sex difference in stroke incidence with increasing age could be due to the higher mortality rate for males than for females(12). Furthermore, people live into an old age stroke-free may represent a more advantageous population. Males who survive to old ages are healthier and have a lower risk of stroke than males have died. Consequently, stroke incidence rate is lower for males than females at older ages. This selective survival can manifest the sex-stroke relationship $(13,14)$, and thus, introduces survivor bias when some unobserved determinants of survival (or mortality) are also associated with stroke. However, existing studies of stroke incidence often restricted to individuals who were alive(9, 10, 15-17). The sex-stroke relationship found might not reflect the casual effect of sex on stroke and could be subject to selection bias, especially when the study sample consisted of old people. Therefore, the degree to which sex disparities in stroke are due to social or biological influences, as opposed to manifestation (an artifact) of selective survival is not well understood.

The objective of this investigation was to evaluate the role of selective survival in explaining the sex disparities in first stroke incidence from mid-life until very old age. We adopted a simulation approach to assess the magnitude of the potential survival bias under several causal scenarios.

\section{Methods}

We simulated a cohort of 100,000 (50,000 male and 50,000 female) individuals who were followed from birth to 95y (or death, whichever occurred first). The mortality rates for age bands 0-1y, 1-5y and every 5y from 5 to $95 y$ were obtained from the US 1959-1961 birth cohort for white males and females(12).

We assumed the first-ever stroke occurred from age 45y. The marginal incidence rates for first stroke between 45-95y were calibrated using the $10 y$ ischaemic stroke incidence rates from the Oxford Vascular Study(10). The rates were adjusted by an increase of $\sim 50-100 \%$ for each sex and age band in our simulation, as the Oxford Vascular Study members were less deprived(18) and had a lower risk of stroke than the rest of England(19). Details about the adjustments are provided in the Supplementary materials (A1).

To quantify the survivor bias, we generated data under the null hypothesis that the male-female difference in first-ever stroke incidence remained 5 per 1000 person-year across age bands from 45 to $95 y$. Any deviation in the observed sex difference from 0.005 person-year reflected survivor bias. We allowed incident stroke to be associated with increased hazard of death within the same or subsequent age band(s), i.e. the effect of stroke history. We assumed that there existed an unobserved and time-invariant construct $U$ that influenced the risk of stroke and/or survival (e.g. genetic variant or lifestyle factors), and no other 
confounders or mediators in the sex-stroke causal pathways (Figure 1). We explored four causal scenarios according to the relations between $U$, incident stroke and survival for males and females to access sex disparities in stroke incidence in each situation considering the mortality rates at different life stages.

\section{Causal scenarios}

In all four causal scenarios (Figure 1), the mortality rate was higher in males than females, especially during early and middle life(12) consistent with the UK national statistics(20). We assumed that stroke incidence rates were also higher for males than females. In addition, the hazard ratios (HR) for mortality associated with instantaneous stroke and stroke history were both set to be the same across all causal scenarios.

In the simulated cohort, $U$ was generated from a standard normal distribution for each individual. The effect of $U$ on incident stroke was the same in all four scenarios $(H R=1.5)$, while its effect on survival (or mortality) varied (Figure 1). Details about the simulation parameters are provided in Supplementary materials (A2).

In causal scenario $A, U$ did not influence mortality $(H R=1.0)$. In scenarios $B$ to $D, U$ had a direct effect on mortality. In scenario $B, U$ influenced mortality with the same magnitude for males and females $(H R=1.5)$. In scenario $C, U$ influenced mortality for males $(H R=1.5)$, but not females $(H R=1.0)$. In scenario $D$, the influence of $U$ on mortality was greater for males $(H R=2.5)$ than females $(H R=1.5)$. As $U$ was positively associated with stroke and/or survival, individuals with larger values of $U$ were more likely to die, resulting in a biased sample consisting of those with smaller values of U. Therefore, the distribution of $U$ would provide insights into the magnitude of survivor bias.

\section{Data simulation and analyses}

Under each scenario, we generated 1000 datasets, each consisted of 100,000 individuals and were followed to $95 \mathrm{y}$. In each simulated dataset, survival data were generated for each age band (0-1y, 1-5y and every $5 y$ thereafter) according to a piecewise Cox proportional hazard model:

$$
H_{i, t}^{\text {survival }}=\operatorname{survival}_{0, t} \cdot \exp \left\{1, t \cdot \text { gender }_{i}+_{2} \cdot U_{i}+3 \cdot \text { gender }_{i} \bullet U_{i}+{ }_{4} \bullet \text { strokehistory }_{i, t}\right\}
$$

1

where $H_{i, t}^{\text {survival }}$ denotes the survival hazard for individual $i$ in band $t, \delta$, $t_{t}$ ivival denotes the baseline survival hazard and $1, t$ denotes the sex difference in survival in band $t$ (same for all individuals); 2 and 3 denote the effect of $U$ and interaction between $U$ and sex on survival respectively; 4 represents the influence of stroke history on survival.

We first generated the survival hazard $H_{i, t}^{\text {survival }}$, and then survival time $T_{i, t}^{\text {survival }}$ by an inverse variable transformation under the exponential failure time model(21). If $T_{i, t}^{\text {survival }}$ was greater than the interval length, individual $i$ survived band $t$ and the simulation continued in the next band $(t+1)$, otherwise, individual $i$ died in this band $(t)$.

Similarly, the first incident stroke (after 45y) was generated according to the piecewise Cox proportional hazard model:

$$
H_{i, t}^{\text {stroke }}=\text { Stroke }_{0, t} \exp \left\{1 \cdot \text { gender }_{i}+_{2} \cdot U_{i}\right\}
$$

2

where $H_{i, t}^{\text {stroke }}$ denotes the stroke hazard for individual $i$ in band $t, s, t$ troke denotes the baseline stroke hazard in band $t, 1$ (fixed at log $(0.005)$ ) represents the male-female difference in stroke incidence (i.e., 5 per 1000 person-year for all age bands); 2 denotes the effect of $U$ on first incident stroke. Similar to the survival model (1), we first generated the stroke hazard $H_{i, t}^{\text {stroke }}$, and then the time to the first incident stroke $T_{i, t}^{\text {stroke }}$ by an inverse variable transformation. Conditional on individual $i$ surviving previous band $(t-1)$, if $T_{i, t}^{\text {stroke }}$ was $<5 \mathrm{y}$, individual $i$ experienced the first incident stroke, otherwise, he/she survived the band stroke-free. Details about the data generating process are provide in Supplementary materials (A3).

Once the survival and stroke data were simulated, we derived the cumulated death and survival rates and stroke incidence rates for males and females in each age band from $45 y$ under four causal scenarios. The stroke incidence rate during a specific age band was calculated as the total number of events (i.e. first incident stroke) divided by the total person-year at risk. Individuals at risk refer to those who were alive and had not experienced the first incident stroke at the beginning of the age band.

All metrics were derived for each dataset, and then averaged over all datasets under each causal scenario. Data simulation and analyses were performed in $\mathrm{R}$ (version 3.6.1).

\section{Results}

In our simulated cohort, median survival time was $77.5 y$ for females and $70 y$ for males. For females, cumulative survival to ages $45,55,65,75$, and $85 y$ differed little under four causal scenarios (Figure 2). Percentages of females surviving to all ages were only slightly lower under scenarios $B$ and $D(U$ influenced mortality, $H R=1.5$ ) compared to those under scenarios $A$ and $C$ (i.e. $U$ did not influence mortality). For males, cumulative survival differed little under scenarios $A, B$ and $C(H R=1.0,1.5$ and 1.5 respectively) and was lower under scenario $D$ (U had a greater effect on mortality than scenarios $A-C, H R=2.5)$. Under scenario $D, 86.4 \%, 74.9 \%, 55.0 \%, 29.8 \%$, and $8.8 \%$ of males survived to $45,55,65,75$, and 85 y respectively, compared to $90.5 \%, 81.3 \%, 63.9 \%, 38.4 \%$, and 12.4\% under scenario A. Similar patterns were seen for cumulative mortality rates by sex and scenario (Supplementary Figure A4.1 and Table A4.1). 


\section{Distribution of $\mathrm{U}$}

Figure 3 illustrates the mean value of $U$ for individuals at risk for the first incident stroke (i.e. were alive and free of stroke at the beginning of each age band) under each causal scenario. For scenario A (with minimum bias), mean value of U remained 0 (pre-specified mean in simulation) until $50 \mathrm{y}$. It declined slightly to below 0 thereafter as individuals with smaller $U$ values were less likely to have a stroke and more likely to remain stroke free at older ages. The reduction was slightly greater in males due to their greater risk for stroke. For scenarios B and C, mean values for males dropped substantially after $45 y$ since $U$ was positively associated with both stroke and death; for females, mean value dropped more rapidly in scenario B (U was associated with both stroke and death) compared to scenario C ( $U$ was associated with stroke only). Under scenario $D$, mean value of $U$ dropped to below 0 at an earlier age, more rapidly thereafter, especially for males due to their strong association with death $(\mathrm{HR}=2.5)$. Thus, survivor bias was introduced when $\mathrm{U}$ influenced both stroke and survival, was greater when the influence was greater for males. Males at risk for stroke at older ages had smaller U values than females, thus were less likely to have stroke (Figure 3). Sex-specific mean values of $U$ at all ages under four causal scenarios are provided in Supplementary Table A4.3.

\section{Stroke incidence rate}

Figure 4 shows the incidence rate for the first incident stroke in 10-year age band from 45 to $95 y$. Under scenario A, the male-female difference in stroke incidence rate was close to the pre-specified 5 per 1000 person-year (no survivor bias) at most ages, became smaller and was biased downwards slightly after $80 y$. Although $U$ did not directly affect mortality, there still existed small survivor bias at old age due to the indirect effect of $U$ on death (via stroke). For example, stroke was more prevalent in males. Individuals with smaller $\mathrm{U}$ were less likely to have stroke, and more likely to survive (since stroke increases risk of death). Under scenario B, the association between $U$ and mortality had the same magnitude in males and females. The male-female difference in stroke incidence was close the pre-specified value until age 75y, reduced to 0 (no sex difference) by 90-95y (Figure 4).

Survivor bias was greater for scenarios $\mathrm{C}$ and $\mathrm{D}$ when there was an interaction effect between $\mathrm{U}$ and sex on mortality. Under scenario $\mathrm{C}(\mathrm{U}$ affected survival only in males), the difference declined slightly from 45 to $65 y$, rapidly from $75 y$, and reversed at age $80-85 y$. Under scenario D (greater effect of $U$ on mortality for males than females: HR=2.5 vs 1.5), the male-female difference reduced throughout adult life, more rapidly after $65 \mathrm{y}$, and reversed earlier at $\sim 80 \mathrm{y}$. For example, compared to females, stroke incidence rate in males was 5 per 1000 person-year greater at $45 y$, but was 9 per 1000 person-year lower at $95 y$. The difference dropped faster in scenario $C$ than scenario $D$ after $85 y$.

Figure 5 shows the male-female difference across age for four scenarios. Since any deviation from the pre-specified 5 per 1000 person-year can be depicted as survivor bias, scenario $A$ had the minimum bias, while scenarios $C$ and $D$ had the highest bias. Our observation about the survivor bias of different magnitudes under scenarios A-D were consistent with the differential distributions of $U$ (Figure 3). Detailed simulation results in terms of the sex-specific stroke incidence rate at all age bands under causal scenarios A-D are provided in Supplementary Table A4.2.

\section{Additional Causal Scenarios}

Simulations under additional causal scenarios $\mathrm{E}$ and $\mathrm{F}$ were conducted to validate our findings. In causal scenario $\mathrm{E}$, the $\mathrm{HR}$ of $\mathrm{U}$ on death was 2.5 for males and 1.0 for females; while in causal scenario $F$, the respective HRs were 1.0 and 1.5. In scenario $E$, survivor bias was even greater than in scenarios $C$ and $D$ : the direction of the male-female difference in stroke incidence reversed at 75y (earlier than 80-85y in scenario C and 75-80y in scenario D); and the malefemale difference reduced to -18 (per 1000 person-year) at $95 y$, compared to -12 in scenario $C$ and -9 in scenario D. For causal scenario F, however, since $U$ affected death in females only, the male-female difference in stroke incidence was biased upwards and increased from the pre-specified 5 (per 1000 personyear) at 45y to 18 (per 1000 person-year) at 95y (Supplementary Figures A5.1, A5.2 and A5.3).

\section{Discussion}

In our simulated cohort, males were observed to have a higher stroke incidence than females in most adult life, but the male-female difference attenuated in late life. The reduction was attributable to selective survival. Moderate bias was observed when there was an unobserved construct $U$ (i.e. common risk factors for stroke and survival) and the effect of $U$ on the hazard of mortality was the same for males and females. Large survivor bias was found when the magnitude of the effect differed by sex.

The sex disparities in stroke incidence have been reported in many studies. In the Oxford Vascular Study where 91,106 individuals were followed from 2002 to 2005 (10), the relative risk (RR) of ischemic stroke for males (vs females) decreased from 1.45 before age $65 y$ to 1.0 after $65 y$. In a population-based study of 15,739 individuals in Scotland during 1986-2005(21), the RR of first stroke hospitalization for males (vs females) decreased from 1.54 at 55-65y to 1.06 at age $\geq 85 y$ after adjusting for years of admission and socioeconomic deprivation. In the Rotterdam study which followed 7721 participants who were free of stroke at baseline for an average of six years, males had higher incidence rates for hospitalized stroke than females between 55-85y, and lower rates after $85 y(15)$. In addition, a national representative study in Spain found that males below 75y had higher rates of acute episodes of stroke, and lower rates after $75 y$ compared to females(17). However, none of the above-mentioned studies considered the issue of selective survival and therefore, the results might be subject to survivor bias. The sex disparities in stroke incidence reported could be over-estimated, especially studies including individuals of advanced ages because of their high death rate.

Few studies have investigated the magnitude of survivor bias in stroke epidemiology. One study investigated the sizes of survivor bias in estimating the difference in stroke incidence between White and Black adults in the US using a simulation approach(22). Their findings suggest that selective survival explains the age attenuation of racial inequalities in stroke incidence. Our simulated cohort had a balanced male-to-female ratio. We explored comprehensive causal scenarios based on the unobserved construct $U$ that affected survival of males and females at various magnitudes, and thus, provides an intuitive guide for assessing the size of survivor bias in the sex inequalities of stroke incidence.

Page $4 / 10$ 
The issue of survivor bias applies to other cardiovascular outcomes. For example, age-adjusted stroke mortality was reported to be $13 \%$ lower in black females than black males in the US according to the WONDER database and the sex-differences decreased with age after 55y(7). In a global assessment of aging during 1980-2010, mortality rates from cardiovascular diseases were higher in males than females, and this difference decreased with age(23). Similar patterns of differences in age-specific estimates have been reported for the associations of high BMI(24) and blood pressure(25) with mortality. The sex inequality was also reported in the case fatality, post-acute care and recovery of stroke(11, 26-33). Biologically, reasons for sex disparities in cardiovascular diseases are complex. It has been suggested that sex steroid hormones may partly explain the higher risk of stroke in males than females during most adulthood. However, limited human studies evaluating the effect of hormones on stroke, and studies assessed the impact of estrogen therapy on cardiovascular outcomes, including stroke among postmenopausal women revealed inconsistent findings(34-37). Therefore, understanding the true sex disparities in stroke epidemiology have important clinical and public health significance.

There are strengths and limitations associated with our simulation study. The survival and stroke rates by sex and age band were calibrated using data from real-world studies to make the hypothetical cohort more "realistic". According to clinical observations, we allowed both instantaneous and history of stroke to be associated with death. Therefore, survivor bias under each causal scenario can be compared with the true value. To better illustrate the effect of selective survival, we assumed that the case-fatality rate and the effect of stroke history on death were equal for males and females, which may not always be true in some clinical settings and for some types of stroke(26). However, this has little impact on our findings since the marginal all-cause mortality was calibrated with respect to the US 1959-61 life table.

In summary, males had higher stroke incidence overall, but the male-female difference attenuated with age (or even reversed) because of selection bias. While males have a higher mortality rate than females, males survive to older age may represent a more advantageous group with a lower risk for incident stroke compared to females. With the growing ageing population, understanding the extent to which the age attenuation of sex disparities represents the elimination of risk factors in males or survivor bias will inform appropriate stroke prevention, treatment and care for females and males. Our simulated birth cohort shows that the magnitude of survivor bias is determined by the unobserved common risk factors for stroke and survival, especially the effect of their interactions with sex on survival. Appropriate statistical analyses are crucial for disentangling the sex inequalities in stroke (as well as other clinical outcomes) from the artifact of selective survival.

\section{Declarations}

Ethics approval and consent to participate: For this simulation study, data were completely simulated, which did not require approval from the ethics committee or consent from participants. All methods were carried out in accordance with relevant guidelines and regulations. The mortality rates by age group used in our simulation were obtained from the US National Centre for Health Statistics and are publicly available from the website:

https://www.cdc.gov/nchs/data/dvs/lewk3_2006.pdf.

Consent for publication: Not applicable.

Availability of data and materials: Methods of data simulation are included within the article. The R-code for simulation is provided in online supplementary materials. The simulated datasets analysed during the current study are available from the corresponding author on reasonable request.

Competing interests: The authors declare that they have no competing interests.

Funding: XL is supported by the Shanghai Sailing Program (19YF1402900) and the General Projects of Shanghai Science and Technology Commission (21ZR1405000). Its contents are solely the responsibility of the authors and do not necessarily represent the official view of Shanghai Commission of Science and Technology.

Authors' contributions: $\mathrm{LL}$ and $\mathrm{XL}$ conceptualized the study and designed the analytical strategy. $\mathrm{XL}$ performed the analysis. $\mathrm{LL}$ and $\mathrm{XL}$ wrote the manuscript. Both authors have read and approved the final manuscript.

Acknowledgements: The authors would like to thank Mingyue Gao for her help to produce some of the figures in this paper.

Authors' information: ${ }^{1}$ School of Data Science, Fudan University, Shanghai, China. ${ }^{2}$ University College London Great Ormond Street Institute of Child Health, 30 Guilford Street, London, UK. WC1N 1EH.

\section{References}

1. The top 10 causes of death. WHO fact sheets. 2020 [Available from: https://www.who.int/news-room/fact-sheets/detail/the-top-10-causes-of-death.

2. Caso V, Paciaroni M, Agnelli G, Corea F, Ageno W, Alberti A, et al. Gender differences in patients with acute ischemic stroke. Womens Health (Lond). 2010;6(1):51-7.

3. Girijala RL, Sohrabji F, Bush RL. Sex differences in stroke: Review of current knowledge and evidence. Vasc Med. 2017;22(2):135-45.

4. Appelman Y, van Rijn BB, Ten Haaf ME, Boersma E, Peters SA. Sex differences in cardiovascular risk factors and disease prevention. Atherosclerosis. 2015;241(1):211-8.

5. Madsen TE, Howard VJ, Jimenez M, Rexrode KM, Acelajado MC, Kleindorfer D, et al. Impact of Conventional Stroke Risk Factors on Stroke in Women: An Update. Stroke. 2018;49(3):536-42.

6. Bushnell CD, Chaturvedi S, Gage KR, Herson PS, Hurn PD, Jimenez MC, et al. Sex differences in stroke: Challenges and opportunities. J Cereb Blood Flow Metab. 2018;38(12):2179-91. 
7. Reeves MJ, Bushnell CD, Howard G, Gargano JW, Duncan PW, Lynch G, et al. Sex differences in stroke: epidemiology, clinical presentation, medical care, and outcomes. Lancet Neurol. 2008;7(10):915-26.

8. Appelros P, Stegmayr B, Terent A. Sex differences in stroke epidemiology: a systematic review. Stroke. 2009;40(4):1082-90.

9. Lofmark U, Hammarstrom A. Evidence for age-dependent education-related differences in men and women with first-ever stroke. Results from a community-based incidence study in northern Sweden. Neuroepidemiology. 2007;28(3):135-41.

10. Rothwell PM, Coull AJ, Silver LE, Fairhead JF, Giles MF, Lovelock CE, et al. Population-based study of event-rate, incidence, case fatality, and mortality for all acute vascular events in all arterial territories (Oxford Vascular Study). Lancet. 2005;366(9499):1773-83.

11. Howard VJ, Cushman M, Pulley L, Gomez CR, Go RC, Prineas RJ, et al. The reasons for geographic and racial differences in stroke study: objectives and design. Neuroepidemiology. 2005;25(3):135-43.

12. Arias E. United States life tables, 2006. Natl Vital Stat Rep. 2010;58(21):1-40.

13. Markides KS, Machalek R. Selective survival, aging and society. Arch Gerontol Geriatr. 1984;3(3):207-22.

14. Sealy-Jefferson S, Wing JJ, Sanchez BN, Brown DL, Meurer WJ, Smith MA, et al. Age- and ethnic-specific sex differences in stroke risk. Gend Med. 2012;9(2):121-8.

15. Hollander M, Koudstaal PJ, Bots ML, Grobbee DE, Hofman A, Breteler MM. Incidence, risk, and case fatality of first ever stroke in the elderly population. The Rotterdam Study. J Neurol Neurosurg Psychiatry. 2003;74(3):317-21.

16. Lewsey JD, Gillies M, Jhund PS, Chalmers JW, Redpath A, Briggs A, et al. Sex differences in incidence, mortality, and survival in individuals with stroke in Scotland, 1986 to 2005. Stroke. 2009;40(4):1038-43.

17. Vega T, Zurriaga O, Ramos JM, Gil M, Alamo R, Lozano JE, et al. Stroke in Spain: epidemiologic incidence and patterns; a health sentinel network study. J Stroke Cerebrovasc Dis. 2009;18(1):11-6.

18. Ministry of Housing, Communities and Local Government, UK. Index of Deprivation 2004 - Average IMD Rank 2014 [Available from: https://data.gov.uk/dataset/1cc8987b-ae6c-49a9-a593-ed5225579f43/index-of-deprivation-2004-average-imd-rank.

19. Rothwell PM, Coull AJ, Giles MF, Howard SC, Silver LE, Bull LM, et al. Change in stroke incidence, mortality, case-fatality, severity, and risk factors in Oxfordshire, UK from 1981 to 2004 (Oxford Vascular Study). Lancet. 2004;363(9425):1925-33.

20. ONS. UK Office for national statistics National life tables - life expectancy in the UK: 2017 to 2019 (2020 release) 2020 [Available from: https://www.ons.gov.uk/peoplepopulationandcommunity/birthsdeathsandmarriages/lifeexpectancies/datasets/nationallifetablesunitedkingdomreferenci

21. Hu P, Tsiatis AA, Davidian M. Estimating the parameters in the Cox model when covariate variables are measured with error. Biometrics. 1998;54(4):140719.

22. Mayeda ER, Banack HR, Bibbins-Domingo K, Zeki Al Hazzouri A, Marden JR, Whitmer RA, et al. Can Survival Bias Explain the Age Attenuation of Racial Inequalities in Stroke Incidence?: A Simulation Study. Epidemiology. 2018;29(4):525-32.

23. Bots SH, Peters SAE, Woodward M. Sex differences in coronary heart disease and stroke mortality: a global assessment of the effect of ageing between 1980 and 2010. BMJ Glob Health. 2017;2(2):e000298.

24. Stevens J, Cai J, Pamuk ER, Williamson DF, Thun MJ, Wood JL. The effect of age on the association between body-mass index and mortality. N Engl J Med. 1998;338(1):1-7.

25. Lewington S, Clarke R, Qizilbash N, Peto R, Collins R, Prospective Studies C. Age-specific relevance of usual blood pressure to vascular mortality: a metaanalysis of individual data for one million adults in 61 prospective studies. Lancet. 2002;360(9349):1903-13.

26. Niewada M, Kobayashi A, Sandercock PA, Kaminski B, Czlonkowska A, International Stroke Trial Collaborative G. Influence of gender on baseline features and clinical outcomes among 17,370 patients with confirmed ischaemic stroke in the international stroke trial. Neuroepidemiology. 2005;24(3):123-8.

27. Thorvaldsen P, Asplund K, Kuulasmaa K, Rajakangas AM, Schroll M. Stroke incidence, case fatality, and mortality in the WHO MONICA project. World Health Organization Monitoring Trends and Determinants in Cardiovascular Disease. Stroke. 1995;26(3):361-7.

28. Olsen TS, Dehlendorff C, Andersen KK. Sex-related time-dependent variations in post-stroke survival-evidence of a female stroke survival advantage. Neuroepidemiology. 2007;29(3-4):218-25.

29. Neyer JR, Greenlund KJ, Denny CH, Keenan NL, Casper M, Labarthe DR, et al. Prevalence of stroke-United States, 2005. Centers for Disease, Control Prevention. MMWR Morb Mortal Wkly Rep. 2007;56(19):469-74.

30. Glader EL, Stegmayr B, Norrving B, Terent A, Hulter-Asberg K, Wester PO, et al. Sex differences in management and outcome after stroke: a Swedish national perspective. Stroke. 2003;34(8):1970-5.

31. Di Carlo A, Lamassa M, Baldereschi M, Pracucci G, Basile AM, Wolfe CD, et al. Sex differences in the clinical presentation, resource use, and 3-month outcome of acute stroke in Europe: data from a multicenter multinational hospital-based registry. Stroke. 2003;34(5):1114-9.

32. Holroyd-Leduc JM, Kapral MK, Austin PC, Tu JV. Sex differences and similarities in the management and outcome of stroke patients. Stroke. 2000;31(8):1833-7.

33. Cavallari LH, Helgason CM, Brace LD, Viana MA, Nutescu EA. Sex difference in the antiplatelet effect of aspirin in patients with stroke. Ann Pharmacother. 2006;40(5):812-7.

34. Hulley S, Grady D, Bush T, Furberg C, Herrington D, Riggs B, et al. Randomized trial of estrogen plus progestin for secondary prevention of coronary heart disease in postmenopausal women. Heart and Estrogen/progestin Replacement Study (HERS) Research Group. JAMA. 1998;280(7):605-13.

35. Viscoli CM, Brass LM, Kernan WN, Sarrel PM, Suissa S, Horwitz RI. A clinical trial of estrogen-replacement therapy after ischemic stroke. N Engl J Med. 2001;345(17):1243-9. 
36. Wassertheil-Smoller S, Hendrix SL, Limacher M, Heiss G, Kooperberg C, Baird A, et al. Effect of estrogen plus progestin on stroke in postmenopausal women: the Women's Health Initiative: a randomized trial. JAMA. 2003;289(20):2673-84.

37. Hendrix SL, Wassertheil-Smoller S, Johnson KC, Howard BV, Kooperberg C, Rossouw JE, et al. Effects of conjugated equine estrogen on stroke in the Women's Health Initiative. Circulation. 2006;113(20):2425-34.

\section{Figures}

Scenario A

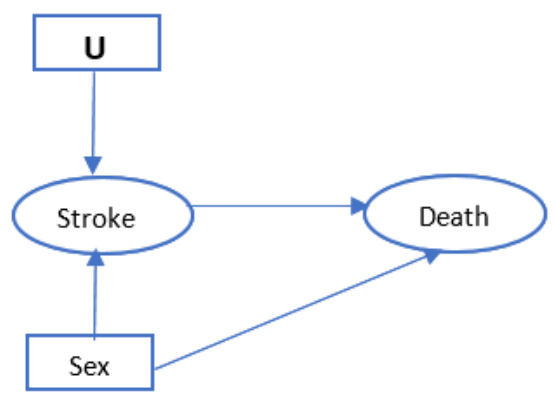

\section{$\underline{\text { Scenario C }}$}

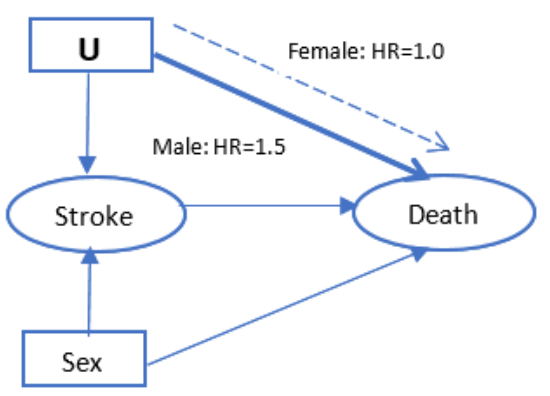

$\underline{\text { Scenario B }}$

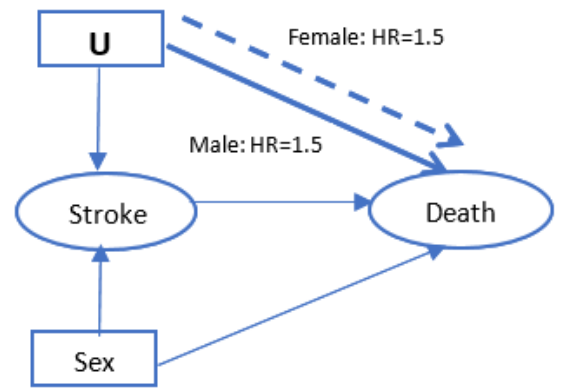

$\underline{\text { Scenario D }}$

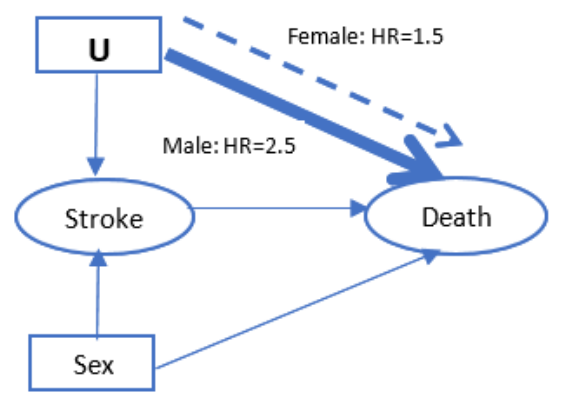

\section{Figure 1}

Four causal scenarios in the simulation study $\dagger$

† Scenario A: $U$ is not associated with death; Scenario B: $U$ is associated with death with a similar magnitude $(H R=1.5)$ for males and females; $S c e n a r i o ~ C: ~ U$ is associated with death for males, not females ( $H R=1.5$ vs 1.0); Scenario $D$ : association of $U$ with death is greater for males than females (HR=2.5 vs 1.5 ). Associations between sex and death, sex and stroke, stroke and death, $\mathrm{U}$ and stroke remain the same across four scenarios. 


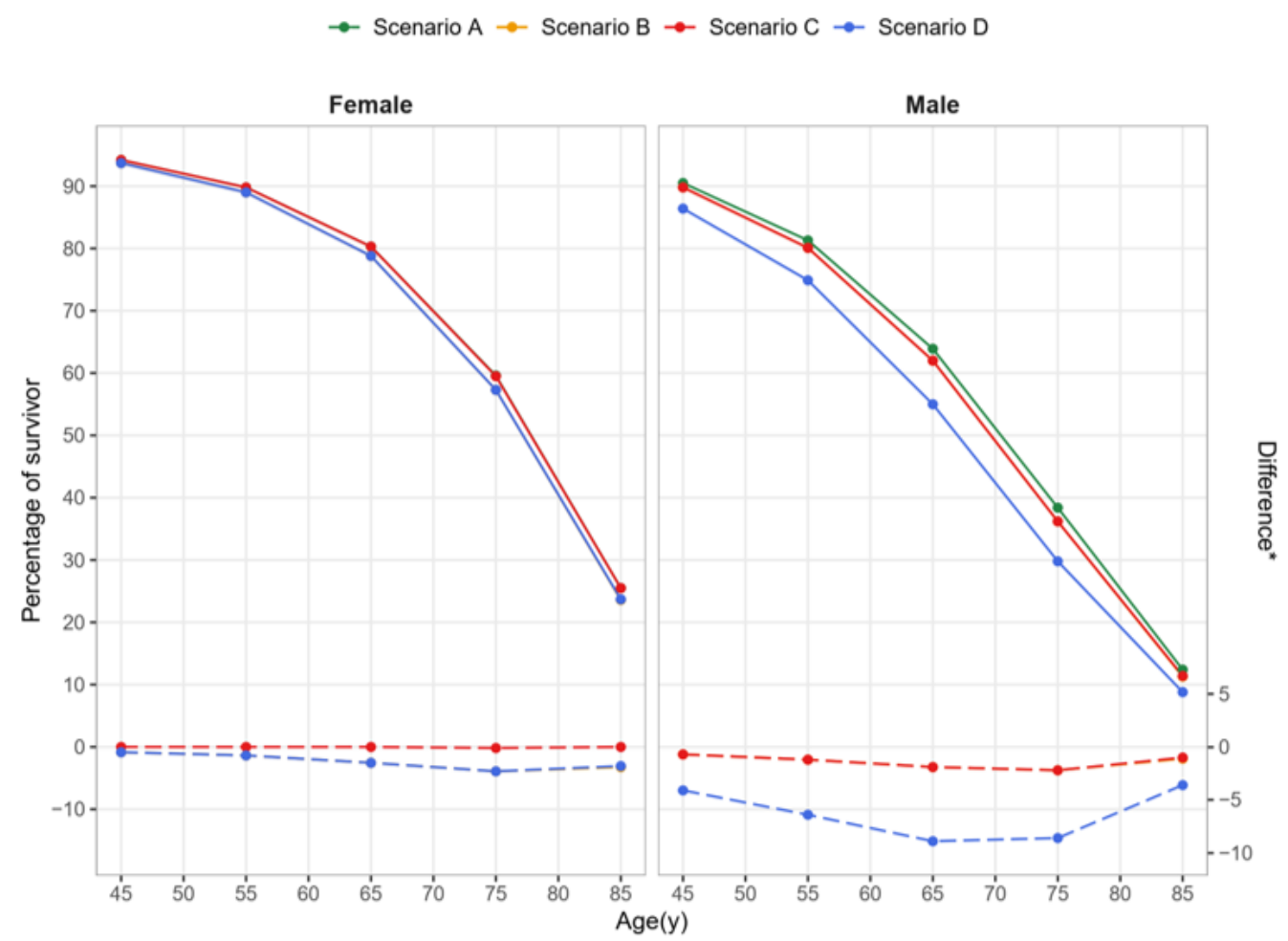

Figure 2

Percentages of females and males surviving to each age in the simulated cohort under four causal scenarios, and differences in percentages between Scenarios B-D and Scenario A*

* Differences in percentages correspond to $y$-axis to the right. As values were similar for some scenarios, for females, red curve represents difference between scenarios $C$ and $A$; blue curve represents differences between scenarios $B / D$ and $A$. For males, red curve represents differences between $B / C$ and $A ;$ blue curve represents difference between $D$ and $A$.

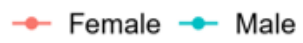

Scenario A

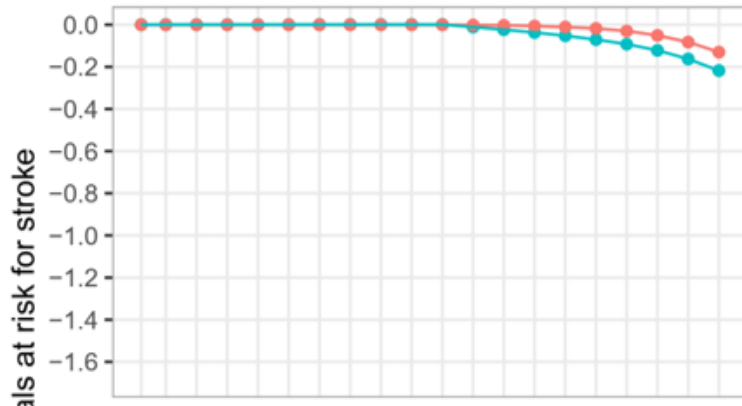

Scenario C

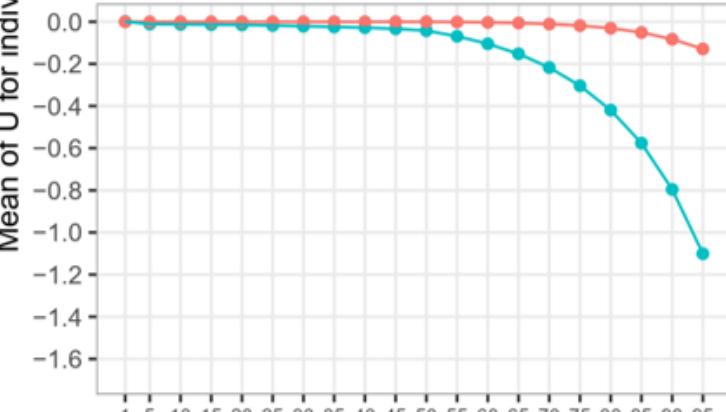

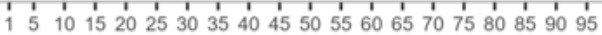

Scenario B

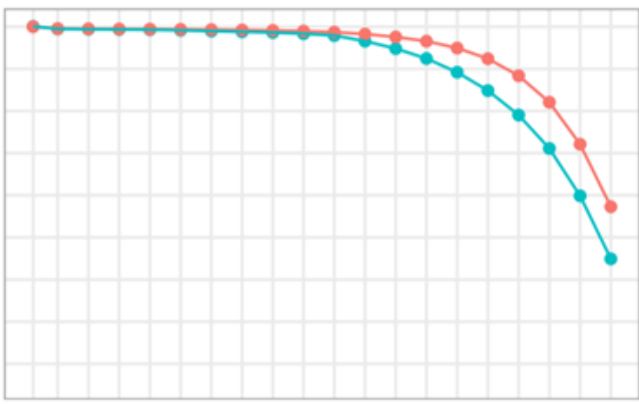

Scenario D

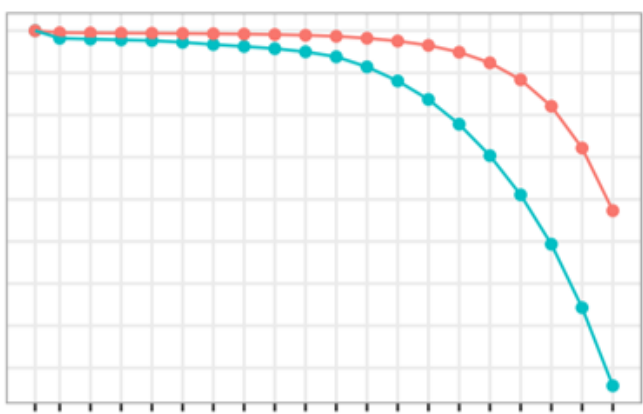

15101520253035404550556065707580859095

Age(y) 
Figure 3

Mean of $U$ for those at risk for the first incident stroke, from birth to age $95 y$ under causal scenarios $A$ to $D$

$$
\rightarrow \text { Female } \rightarrow \text { Male }
$$

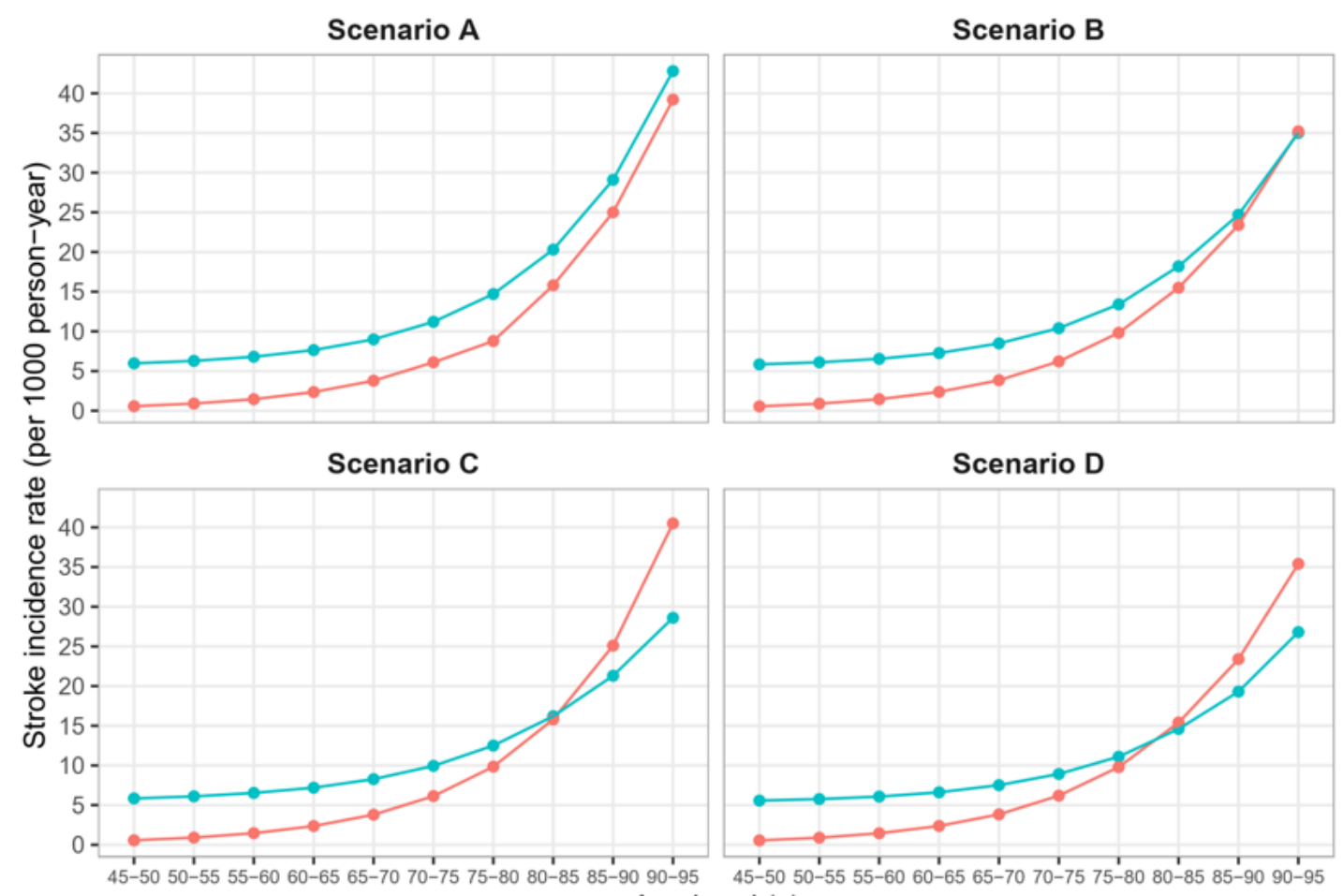

Age band $(y)$

Figure 4

Stroke incidence rate for males and females at $5 y$ age bands in the simulated cohort under four causal scenarios

- Scenario A $\rightarrow$ Scenario B $\rightarrow$ Scenario C $\rightarrow$ Scenario D

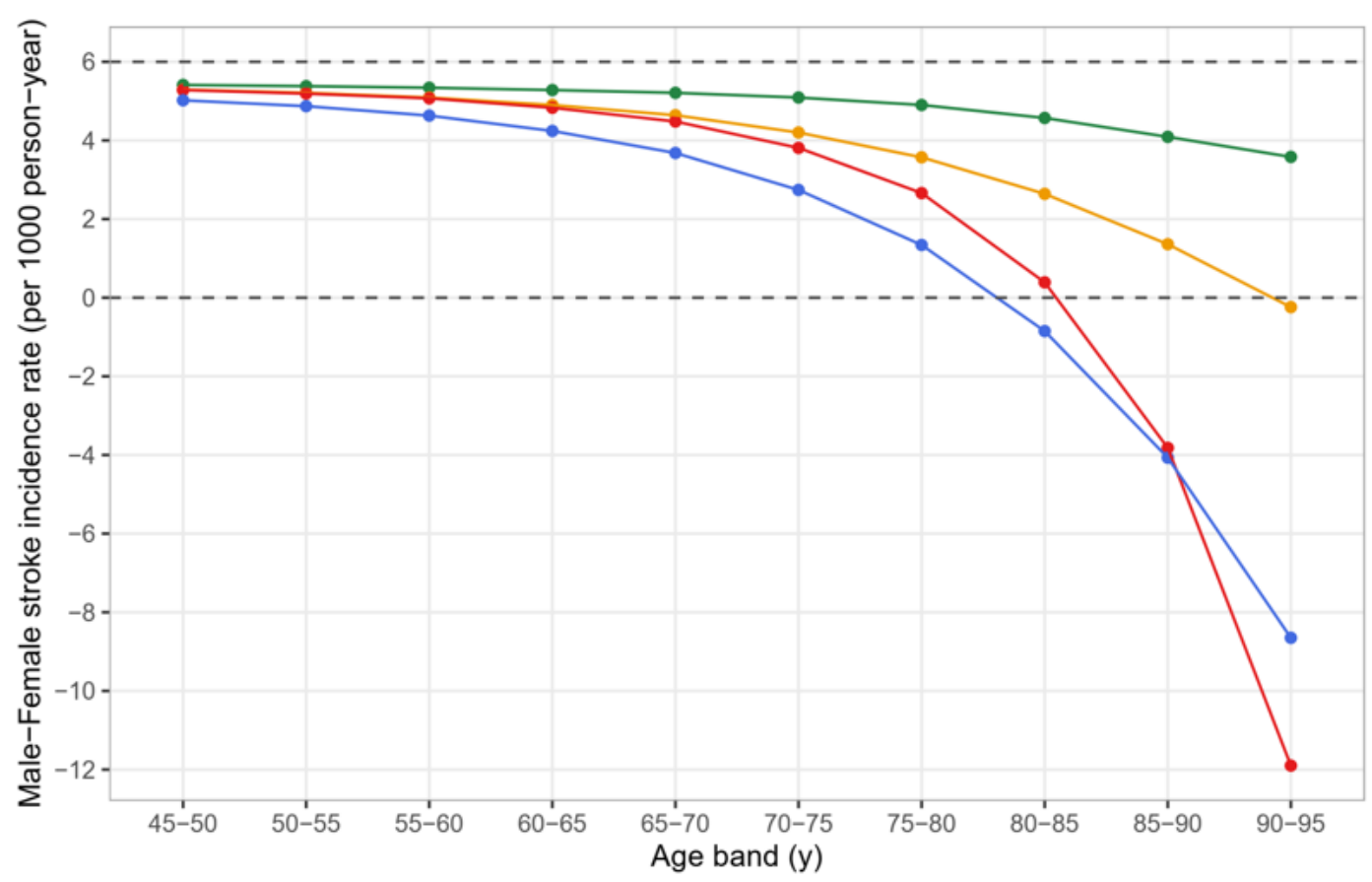

Figure 5 
Difference in stroke incidence rate (per 1000 person-year) between males and females for $5 y$ age bands in the simulated cohort under four causal scenarios.

Pre-specified male - female difference is 5 per 1000 person-year and marked by the black dash line

\section{Supplementary Files}

This is a list of supplementary files associated with this preprint. Click to download.

- Supplementary28Dec21submitted.docx 